\title{
Decentralized Robust Energy and Reserve Co-optimization for Multiple Integrated Electricity and Heating Systems
}

\author{
Tan, Jin; Wu, Qiuwei; Wei, Wei; Liu, Feng; Li, Canbing; Zhou, Bin
}

Published in:

Energy

Link to article, DOI:

10.1016/j.energy.2020.118040

Publication date:

2020

Document Version

Early version, also known as pre-print

Link back to DTU Orbit

Citation (APA):

Tan, J., Wu, Q., Wei, W., Liu, F., Li, C., \& Zhou, B. (2020). Decentralized Robust Energy and Reserve Cooptimization for Multiple Integrated Electricity and Heating Systems. Energy, 205, [118040].

https://doi.org/10.1016/j.energy.2020.118040

\section{General rights}

Copyright and moral rights for the publications made accessible in the public portal are retained by the authors and/or other copyright owners and it is a condition of accessing publications that users recognise and abide by the legal requirements associated with these rights.

- Users may download and print one copy of any publication from the public portal for the purpose of private study or research.

- You may not further distribute the material or use it for any profit-making activity or commercial gain

- You may freely distribute the URL identifying the publication in the public portal 


\title{
Decentralized Robust Energy and Reserve Co-optimization for Multiple
}

\section{Integrated Electricity and Heating Systems}

\author{
Jin Tan ${ }^{\mathrm{a}}$, Qiuwei $\mathrm{Wu}^{\mathrm{a}, *}$, Wei Wei ${ }^{\mathrm{b}}$, Feng Liu ${ }^{\mathrm{b}}$, Canbing $\mathrm{Li}^{\mathrm{c}}$ and Bin $\mathrm{Zhou}^{\mathrm{d}}$ \\ ${ }^{a}$ Center for Electric Power and Energy, Department of Electrical Engineering, Technical University of Denmark, Kgs. Lyngby, 2800 Denmark \\ b Department of Electrical Engineering, Tsinghua University, Beijing, China, 100084 \\ c Department of Electrical Engineering, Shanghai Jiaotong University, Shanghai, China, 200240 \\ d College of Electrical and Information Engineering, Hunan University, Changsha, China, 410082
}

\begin{abstract}
The growing uncertainty caused by the increasing wind power penetration brings an urgent need of increased reserve capacity to ensure the secure operation of power systems. The interconnection of multiple integrated electricity and heating systems (IEHSs) allows resource sharing among them and the district heating system can provide additional reserves to the electric power system. In this paper, a decentralized robust energy and reserve co-optimization for multiple IEHSs is proposed to handle wind power uncertainty in a more economic and efficient way. In each IEHS, the day-ahead energy and reserve scheduling is formulated as a two-stage adaptive robust optimization, where the feasibility of day-ahead reserve deployment in the real-time operation is guaranteed. Then, a decentralized methodology is developed based on the alternating direction method of multipliers (ADMM) to achieve the synergistic yet independent operation by exchanging tie-line power flows with adjacent IEHSs. In order to accelerate the convergence of the decentralized operation and reduce the communication demand, the improved ADMM with locally adaptive penalty parameters is adopted. Simulation results demonstrate the effectiveness of the proposed decentralized robust energy and reserve optimization scheme in terms of improving the economic efficiency and ensuring the secure operation for IEHSs, as well as preserving information privacy.
\end{abstract}

\section{Highlights}

A decentralized energy and reserve co-optimization for multiple IEHSs is proposed.

The feasibility of reserves is guaranteed with the two-stage robust optimization.

An improved ADMM algorithm with adaptive penalty parameters is adopted.

The economic efficiency of IEHSs is improved and information privacy is preserved.

\section{Keywords}

Adaptive robust optimization, energy and reserve co-optimization, improved ADMM algorithm, integrated electricity and heating system.

\begin{tabular}{|l}
\hline Indices and sets \\
$\Phi_{s}^{\mathrm{N} / \mathrm{G} / \mathrm{WF} / \mathrm{ED}}$ \\
$\Phi_{s}^{\mathrm{BB}}$ \\
$\Phi_{s}^{\mathrm{FB}}$ \\
$\Phi_{s}^{\mathrm{CHP} / \mathrm{EB}}$ \\
$\Phi_{s}^{\mathrm{HN} / \mathrm{HS} / \mathrm{HD}}$ \\
$\Phi_{s}^{\mathrm{S} / \mathrm{R}, \mathrm{pipe}}$ \\
$\Phi_{n}$
\end{tabular}

Set of internal buses/thermal units/wind farms/electric demand in IEHS $s$

Set of boundary buses in IEHS $s$

Set of fictitious boundary buses in IEHS $s$

Set of CHP units/electric boilers in IEHS $s$

Set of heat nodes/heat sources/heat demands in DHS in IEHS $s$

Set of supply/return pipelines in DHS in IEHS $s$

Set of adjacent IEHSs connected to bus $n$ 


\begin{tabular}{|c|c|}
\hline$\Omega_{n}^{\mathrm{CHP} / \mathrm{EB}}$ & Set of CHP units/electrical boilers connected to bus $n$ \\
\hline$\Omega_{n}^{\mathrm{G} / \mathrm{WF} / \mathrm{ED} / \mathrm{N}}$ & Set of thermal units/wind farms/electric demands/internal buses connected to $b$ \\
\hline$\Omega_{j}^{\mathrm{HN} / \mathrm{CHP} / \mathrm{EB}}$ & Set of heat nodes/CHP units/ electrical boilers corresponding to heat source $j$ \\
\hline$\Omega_{n d}^{\mathrm{B} / \mathrm{E}, \mathrm{S} / \mathrm{R}, \mathrm{pipe}}$ & Set of supply/return pipelines beginning/ending at node $n d$ \\
\hline$T$ & Set of hours \\
\hline $\mathrm{DA} / \mathrm{RT}$ & Day-ahead stage/Real-time stage \\
\hline \multicolumn{2}{|l|}{ Parameters } \\
\hline$C_{s}^{D A, E N / R C}$ & Cost of day-ahead energy/reserve in IEHS $s$ \\
\hline$C_{s}^{P E / R T}$ & Penalty term/Cost of real-time redisptach \\
\hline$c_{h, t}^{E / H, \mathrm{CHP}}$ & Power/heat production cost rates offered by CHP units \\
\hline$c_{g, t}^{\mathrm{G}}$ & Production cost rate offered by non-CHP thermal units \\
\hline$c_{h, t}^{\mathrm{R}}, c_{g, t}^{\mathrm{R}}$ & Cost rates offered by CHP units/non-CHP thermal units for reserve capacity \\
\hline$c_{l}^{\text {shed }}$ & Cost rate for load shedding \\
\hline$B_{n m}, f_{n m}^{\max }$ & Susceptance/maximum power of transmission line $\mathrm{nm}$ \\
\hline$P_{w, t}^{\mathrm{wd}}, P_{l, t}^{\mathrm{ED}}$ & Forecasted wind power/Electric demand \\
\hline$R U_{h, t}^{\mathrm{CHP}}, R D_{h, t}^{\mathrm{CHP}}$ & Ramping up/down limit of CHP units \\
\hline$R U_{g, t}^{\mathrm{G}}, R D_{g, t}^{\mathrm{G}}$ & Ramping up/down limit of non-CHP thermal units \\
\hline$P_{h, t}^{\mathrm{CHP}, \max / \min }$ & Maximum/minimum generation of CHP units \\
\hline$P_{g, t}^{\mathrm{G}, \max / \min }$ & Maximum/minimum generation of non-CHP thermal units \\
\hline$P_{i, t}^{\mathrm{EB}, \max }$ & Maximum power consumption of electric boiler \\
\hline$\Delta W_{w, t}^{\max }$ & Maximum deviation of wind power \\
\hline$\eta_{h}^{\mathrm{CHP}}$ & Heat to power coefficient of CHP units \\
\hline$\eta_{i}^{\mathrm{EB}}$ & Power to heat coefficient of electric boiler \\
\hline$c, l_{p}$ & Specific heat capacity of water/Length of pipelines \\
\hline$m^{\mathrm{HS} / \mathrm{HD}}$ & Mass flow rate of heat sources/heat loads \\
\hline$m^{\mathrm{S} / \mathrm{R}, \mathrm{pipe}}$ & Mass flow rate of supply/return pipelines \\
\hline$\tau_{t}^{a}$ & Ambient temperature of pipelines \\
\hline$\rho$ & Penalty parameter in ADMM \\
\hline \multicolumn{2}{|l|}{ Variables } \\
\hline$P_{h, t}^{\mathrm{CHP}}, P_{g, t}^{\mathrm{G}}$ & Day-ahead generation of CHP/ non-CHP thermal units \\
\hline$P_{i, t}^{\mathrm{EB}}$ & Day-ahead electricity consumption of electric boilers \\
\hline$R_{h, t}^{\mathrm{CHP}}, R_{g, t}^{\mathrm{G}}$ & Day-ahead reserve capacity scheduled for CHP/non-CHP thermal units \\
\hline$r_{h, t}^{\mathrm{CHP}, \mathrm{U} / \mathrm{D}}$ & Upward/downward redispatch of CHP units \\
\hline$r_{g, t}^{\mathrm{G}, \mathrm{U} / \mathrm{D}}$ & Upward/downward redispatch of non-CHP thermal units \\
\hline$L_{l, t}^{\text {shed }}$ & Load shedding \\
\hline$\Delta W_{w, t}, W_{w, t}^{\text {sill }}$ & Deviation of wind power/Wind spillage \\
\hline$\delta_{s, n, t}$ & Phase angle of buses in IEHS $s$ \\
\hline $\bar{\delta}_{n, t}$ & Average angle of fictitious boundary buses in adjacent IEHSs \\
\hline$H_{h, t}^{\mathrm{CHP}}, H_{i, t}^{\mathrm{EB}}$ & Heat output of CHP units/electric boilers \\
\hline$H_{h, t}^{\mathrm{CHP}, \mathrm{RT}}$ & Heat output of CHP units in real-time stage \\
\hline$\tau_{n d, t}^{\mathrm{S} / \mathrm{R}, \mathrm{ND}}$ & Temperature of node $n d$ in supply/return networks \\
\hline$\tau_{p, t}^{\mathrm{S} / \mathrm{R}, \text { in }}, \tau_{p, t}^{\mathrm{S} / \mathrm{R}, \text { out }}$ & Mass flow temperature at the inlet/outlet of pipeline in supply/return ne \\
\hline
\end{tabular}


With the increasing renewable energies integrated into power systems, the growing fluctuation and limited predictability pose great challenges to the secure and reliable operation of power systems. Reserve capacity, as a commodity traded in power systems [1], has worked effectively to compensate power imbalances and fluctuations. Due to the increasing renewable generation uncertainty, there is an urgent need of increased reserve and additional flexibility to deal with power imbalances in the system operation. Moreover, a more cost-efficient energy and reserve allocation scheme is desired for the system operation to cope with uncertainties. With the rapid development of the multi-energy system, the electric power system (EPS) and district heating system (DHS) are coupled more tightly and deeply [2]. On the one hand, the joint operation of integrated electricity and heating system (IEHS) enables the DHS to provide additional reserve capacity to the EPS [3]. On the other hand, as an important feature of modern energy system, the increasing interconnection of multiple IEHSs allows the energy and reserve resources to be shared among interconnected energy markets. In this regard, the energy and reserve costs of the whole system could be reduced with the efficient coordination among multiple IEHSs. Accordingly, it is essential to investigate new energy and reserve scheduling schemes and utilize additional reserve capacity in multiple interconnected IEHSs to minimize the overall system operation cost.

Given the high efficiency of coupled EPS and DHS, the joint optimal operation of IEHS has been extensively researched. In order to accommodate more fluctuating renewable energies, many technologies and components are introduced to enhance the flexibility of combined heat and power (CHP) units. In [4] and [5], the thermal dynamic characteristics of the existing DHS, i.e., the heat storage capacity of district heating networks and thermal inertia of buildings, are utilized to decouple the heat demand and CHP heat generation. Hence, the imbalance between the heat generation and consumption can be accepted temporarily. Meanwhile, additional components, such as thermal storage devices [6], heat pumps [7] and electric boilers (EBs) [8], are invested in the DHS such that the heat generation of CHP units is less dependent on heat demands. Considering that the EPS and DHS are operated by different operators, decentralized strategies are adopted to achieve the synergistic operation for the IEHS. A decentralized solution based on alternating direction method of multipliers (ADMM) was adopted in [9] to solve the dispatch model considering heat dynamics. Refs. [10] and [11] proposed decentralized solutions based on Benders decomposition and optimal condition decomposition to achieve the coordination of combined heat and power dispatch.

Based on the aforementioned technologies to improve the CHP units' flexibilities, the DHS can provide certain reserve capacity to the EPS to handle system uncertainties [12]. In [13] and [14], the reserve capacity of CHP units was considered in the energy and reserve optimization of IESs, but CHP units were assumed to provide full reserves without considering the limitation of realtime redispatch constraints. This may result in infeasible real-time redispatch implementation. Therefore, [15] proposed a feasible region model for DHSs accounting for the building thermal inertia, which considers the flexibility of the DHS and can be directly used in the operation dispatch. Ref. [16] presented a regulating region method to properly assess the available reserve capacity of CHP units. However, the reserve capacity allocation in these researches is obtained according to the pre-defined minimum reserve requirements based on operators' experiences. The solution is suboptimal due to the deterministic optimization and may even be infeasible without considering operation constraints in the real-time stage [17]. Therefore, with the large-scale penetration of wind power, it is imperative to develop an effective reserve allocation method adaptive to the uncertainty while taking into account redispatch constraints. Two-stage robust optimization, unlike the probabilistic method requiring accurate probability distribution functions [18] or the stochastic optimization with a heavy computation burden [19], is much briefer taking into account the worstcase realization of uncertainty. In this paper, the energy and reserve scheduling for each IEHS is formulated as a two-stage adaptive robust scheme considering real-time operation constraints, where the real-time adjustments of generators are carried out according to the reserve scheduled in the day-ahead operation.

Moreover, the interconnection of multiple IEHSs allows the interregional resources sharing [20]. As such, the effective energy and reserve coordination among multiple IEHSs can cope with uncertainties better while improving the overall system economic efficiency. The ideal operation for multiple IEHSs is to optimize the energy and reserve by coordinating all IEHSs with a central 
by different entities. It is impractical for a central operator to collect all data of generating units, transmission networks, and loads from different IEHSs. Therefore, a decentralized optimization framework is desired to achieve the synergistic but independent operation for all IEHSs with information privacy protected. According to [23], the decentralized algorithms used in the EPS are typically classified into two categories: one is based on the augmented Lagrangian relaxation and the other one is based on the first-order optimal conditions. Owing to the low amount of data exchange and application simplicity, the ADMM is widely used to solve the multi-area EPS optimization problems in a decentralized manner. In [24], the ADMM algorithm was used to achieve the decentralized multi-area generation unit and tie-line scheduling. Ref. [25] adopted an iterative ADMM scheme to deal with binary variables in the optimal energy flow of multi-area electricity-natural gas systems. Nevertheless, the convergence performance of the ADMM is sensitive to the penalty parameters [26]. In the aforementioned studies, constant penalty parameters are used in the standard ADMM. In order to improve the convergence rate of the decentralized algorithm and decrease the communication demand, variants of ADMM with adaptive penalty parameters should be explored and adopted.

Given this background, a proper energy and reserve co-optimization scheme for multiple IEHSs needs to be investigated in depth and the available reserve capacity in multiple IEHSs should be fully utilized. The reserve allocation determined in previous researches may be suboptimal or even infeasible without considering real-time redispatch constraints. Therefore, this paper proposes an efficient decentralized energy and reserve co-optimization scheme for multiple IEHSs, which improves the overall economic efficiency for the energy and reserve scheduling while preserving the information privacy for each IEHSs. In addition, the wind uncertainty is addressed by the two-stage adaptive robust programming ensuring the feasibility of day-ahead energy and reserve deployment. The contributions of this paper are summarized as follows.

1) A decentralized energy and reserve co-optimization scheme for multiple IEHSs is proposed. By using the additional reserve capacity from the DHS and sharing resources with adjacent IEHSs, the overall cost of the system is reduced and the information privacy of different entities is preserved.

2) The two-stage adaptive robust optimization accounting for real-time operation constraints is developed to accommodate the wind uncertainty for each IEHS, where the feasibility of the day-ahead reserve deployment is guaranteed.

3) An improved ADMM algorithm with adaptive penalty parameters is adopted to increase the convergence rate and solution efficiency of the decentralized operation, as well as reducing the communication demand.

The rest of the paper is organized as follows. Section 2 introduces the structure and operation framework of multiple IEHSs. Section 3 and 4 describe the mathematical model and solution method for the proposed decentralized robust energy and reserve optimization scheme. Section 5 shows the numerical results, followed by the conclusions.

\section{Structure and decentralized operation framework of multiple IEHSs}

The schematic structure of interconnected multiple IEHSs is shown in Fig. 1. In each IEHS, the EPS and DHS are coupled with CHP units and EBs. Electric power and heat are transmitted from the generation side to the demand side through power and district

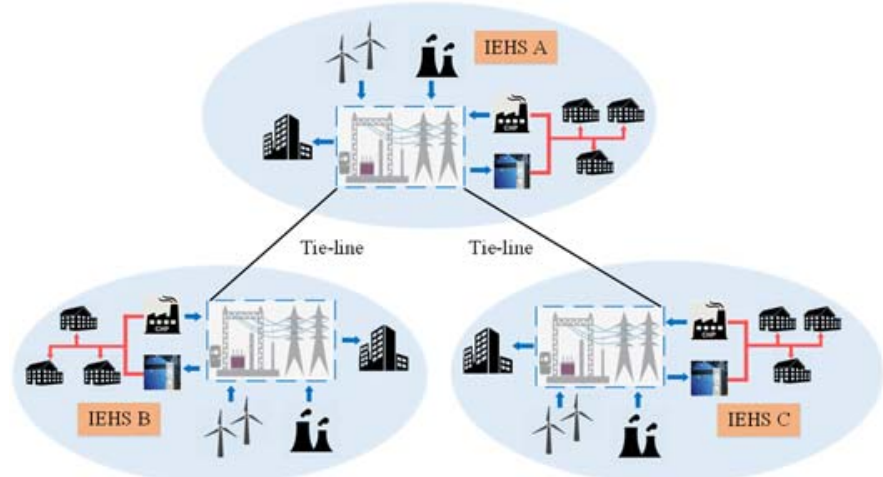


heating networks, respectively. Typically, heat is generated, transferred, and consumed in a local region, whereas the electricity can be transferred over long distances. Thus, in this paper, adjacent IEHSs are only interconnected by electric power tie-lines, as illustrated in Fig. 1.

Ideally, in order to achieve the highest economic efficiency, all IEHSs shall be operated as a whole system with a central operator. However, in reality, different IEHSs are owned by different entities and there is no central operator with access to all the data of the whole system. Thus, the decentralized operation framework is needed to maintain the independent but synergistic energy and reserve scheduling for each IEHS. As shown in Fig. 1, adjacent IEHSs are coupled with tie-lines and the whole system cannot be separated by relaxing the coupling constraints. Thus, fictitious boundary buses, representing the copies of the boundary buses in adjacent IEHSs, are created in each IEHS, which are represented by the dotted lines in Fig. 2. The corresponding phase angles, i.e., $\delta_{A, j}$ and $\delta_{A, l}$ in IEHS A, $\delta_{B, i}$ in IEHS B, and $\delta_{C, k}$ in IEHS C, are also added in the set $\Phi_{\mathrm{e}}^{\mathrm{FB}}$ in each IEHS. Eqs. (1)-(2) represent that the phase angles of copied buses, e.g. $\delta_{A, j}$, are the same as that of the real boundary buses, e.g. $\delta_{B, j}$. The whole system is decomposed into several IEHS subsystems by relaxing (1)-(2), which is shown in Fig. 2.

$\delta_{A, j}=\delta_{B, j}, \delta_{A, l}=\delta_{C, l}$

$\delta_{B, i}=\delta_{A, i}, \delta_{C, k}=\delta_{A, k}$

\section{Mathematical formulation of decentralized robust energy and reserve co-optimization for multiple IEHSs}

In this section, the mathematical model of the decentralized adaptive robust energy and reserve co-optimization for multiple IEHSs to cope with the wind uncertainty is presented.

\subsection{Objective function}

According to the joint market structures such as the PJM market in North American [27], the day-ahead energy and reserve capacity is optimized simultaneously. Taking into account the day-ahead and real-time operation constraints of both the EPS and DHS, the energy and reserve co-optimization problem is cast as a two-stage adaptive robust scheme. In the first stage, the dayahead energy and reserve capacity, as well as the tie-line power flows, are scheduled with the predicted wind power generation. In the second stage, when the wind uncertainty is revealed in the real-time operation, the day-ahead scheduled reserve and generation adjustments are deployed.

In this paper, the objective is to optimize the day-ahead energy and reserve scheduling by minimizing the total system operation cost under the worst-case realization of wind uncertainty while properly utilizing the reserve capacity from the DHS and the shared reserves from interconnected IEHSs. By relaxing the coupling constraints (1)-(2), a weighted penalty term corresponding to the phase angles of boundary buses is introduced in the first stage. After the decomposition, the objective function for each IEHS is formulated as the augmented Lagrangian (3).

$$
\min \left(C_{s}^{\mathrm{DA}, \mathrm{EN}}+C_{s}^{\mathrm{DA}, \mathrm{RC}}+C_{s}^{\mathrm{PE}}+\max _{\Delta W_{w, t}} \min C_{s}^{\mathrm{RT}}\right)
$$




$$
C_{s}^{\mathrm{DA}, \mathrm{EN}}=\sum_{t \in T}\left\{\sum_{h \in \Phi_{s}^{\mathrm{CHP}}}\left(c_{h, t}^{\mathrm{E}, \mathrm{CHP}} P_{h, t}^{\mathrm{CHP}}+c_{h, t}^{\mathrm{H}, \mathrm{CHP}} H_{h, t}^{\mathrm{CHP}}\right)+\sum_{g \in \Phi_{s}^{G}} c_{g, t}^{\mathrm{G}} P_{g, t}^{\mathrm{G}}\right\}
$$

$$
\begin{gathered}
C_{s}^{\mathrm{DA}, \mathrm{RC}}=\sum_{t \in T}\left(\sum_{h \in \Phi_{s}^{\mathrm{CHP}}} c_{h, t}^{\mathrm{R}} R_{h, t}^{\mathrm{CHP}}+\sum_{g \in \Phi_{s}^{G}} c_{g, t}^{\mathrm{R}} R_{g, t}^{\mathrm{G}}\right) \\
C_{s}^{\mathrm{PE}}=\sum_{t \in T} \sum_{n \in \Phi_{s}^{\mathrm{BB}} \cup \Phi_{s}^{\mathrm{EB}}}\left\{\lambda_{s, n, t}\left(\delta_{s, n, t}-\bar{\delta}_{n, t}\right)+0.5 \rho\left\|\delta_{s, n, t}-\bar{\delta}_{n, t}\right\|_{2}^{2}\right\} \\
C_{s}^{\mathrm{RT}}=\sum_{t \in T}\left\{\sum_{h \in \Phi_{s}^{\mathrm{CHP}}} c_{h}^{\mathrm{E}, \mathrm{CHP}}\left(r_{h, t}^{\mathrm{CHP}, \mathrm{U}}-r_{h, t}^{\mathrm{CHP}, \mathrm{D}}\right)+\sum_{g \in \Phi_{s}^{G}} c_{g}^{\mathrm{G}}\left(r_{g, t}^{\mathrm{G}, \mathrm{U}}-r_{g, t}^{\mathrm{G}, \mathrm{D}}\right)+\sum_{h \in \Phi_{s}^{\mathrm{CHP}}} c_{h}^{\mathrm{H}, \mathrm{CHP}}\left(H_{h, t}^{\mathrm{CHP}, \mathrm{RT}}-H_{h, t}^{\mathrm{CHP}}\right)+\sum_{l \in \Phi_{s}^{E L L}} c_{l}^{\mathrm{shed}} L_{l, t}^{\mathrm{shed}}\right\} \\
\bar{\delta}_{n, t}=\left(\sum_{s \in \Phi_{n}} \delta_{s, n, t}\right) /\left|\Phi_{n}\right|
\end{gathered}
$$

142

143

144

145

146

147

148

149

150

151

152

153

154

155

156

157

158

159

160

161

Objective (3) is to minimize the total system operation cost for each IEHS, including the day-ahead energy and reserve cost and real-time redispatch cost. The max-min term in (3) is to calculate the redispatch cost under the worst-case realization of wind uncertainty. Eqs. (4)-(7) represent the day-ahead energy generation cost, reserve allocation cost, penalty term, and the real-time redispatch cost of each IEHS, respectively, (8) is the average phase angle of all boundary buses in IEHS $s$ connected to the bus $n$, and $\left|\Phi_{n}\right|$ is the number of fictitious boundary buses in adjacent IEHSs connected to bus $n$.

\subsection{Day-ahead operation constraints of IEHSs}

The energy and reserve scheduling is implemented in the day-ahead electricity market with the predicted wind power, which is regarded as the first stage and is robust to the wind uncertainty. The day-ahead operation constraints consist of both the EPS and DHS constraints in each IEHS and power flow limits of tie-lines. The detailed formulations are shown as follows.

\subsubsection{Day-ahead operation constraints of EPS}

As shown in Fig. 1, the electricity demand is supplied by CHP units, thermal units and wind farms. The optimization of the EPS is formulated based on the DC power flow model. The day-ahead operation constraints of each EPS are as follows:

$$
P_{h, t}^{\mathrm{CHP}}+R_{h, t}^{\mathrm{CHP}} \leq P_{h, t}^{\mathrm{CHP}, \max }, \forall h \in \Phi_{s}^{\mathrm{CHP}}, \forall t \in T
$$

$P_{h, t}^{\mathrm{CHP}, \min } \leq P_{h, t}^{\mathrm{CHP}}-R_{h, t}^{\mathrm{CHP}}, \forall h \in \Phi_{s}^{\mathrm{CHP}}, \forall t \in T$

$P_{g, t}^{\mathrm{G}}+R_{g, t}^{\mathrm{G}} \leq P_{g, t}^{\mathrm{G}, \max }, \forall g \in \Phi_{s}^{\mathrm{G}}, \forall t \in T$

$P_{g, t}^{\mathrm{G}, \min } \leq P_{g, t}^{\mathrm{G}}-R_{g, t}^{\mathrm{G}}, \forall g \in \Phi_{s}^{\mathrm{G}}, \forall t \in T$

$$
0 \leq R_{h, t}^{\mathrm{CHP}} \leq \min \left(R U_{h}^{\mathrm{CHP}}, R D_{h}^{\mathrm{CHP}}\right), \forall h \in \Phi_{s}^{\mathrm{CHP}}, \forall t \in T
$$

$$
0 \leq R_{g, t}^{\mathrm{G}} \leq \min \left(R U_{g}^{\mathrm{G}}, R D_{g}^{\mathrm{G}}\right), \forall g \in \Phi_{s}^{\mathrm{G}}, \forall t \in T
$$

$$
-R D_{h}^{\mathrm{CHP}} \Delta t \leq P_{h, t}^{\mathrm{CHP}}-P_{h, t-1}^{\mathrm{CHP}} \leq R U_{h}^{\mathrm{CHP}} \Delta t, \forall h \in \Phi_{s}^{\mathrm{CHP}}, \forall t \in T
$$

$$
-R D_{g}^{\mathrm{G}} \Delta t \leq P_{g, t}^{\mathrm{G}}-P_{g, t-1}^{\mathrm{G}} \leq R U_{g}^{\mathrm{G}} \Delta t, \forall g \in \Phi_{s}^{\mathrm{G}}, \forall t \in T
$$




$$
\sum_{h \in \Omega_{n}^{\mathrm{CHP}}} P_{h, t}^{\mathrm{CHP}}+\sum_{g \in \Omega_{n}^{\mathrm{G}}} P_{g, t}^{\mathrm{G}}+\sum_{w \in \Omega_{n}^{\mathrm{WF}}} P_{w, t}^{\mathrm{wd}}-\sum_{i \in \Omega_{n}^{\mathrm{EB}}} P_{i, t}^{\mathrm{EB}}-\sum_{l \in \Omega_{n}^{\mathrm{ED}}} P_{l, t}^{\mathrm{load}}=\sum_{m \in \Omega_{n}^{\mathrm{N}}} B_{n m}\left(\delta_{s, n, t}-\delta_{s, m, t}\right), \forall n \in \Phi_{s}^{\mathrm{N}}, \forall t \in T
$$

164

165

166

167

168

169

170

171

172

173

174

175

176

177

178

where (9)-(12) are generation capacity constraints of CHP units and thermal units considering reserve capacity, respectively, (13)(14) indicate the scheduled reserve capacity of generating units should be smaller than corresponding ramping rate, (15)-(16) are the ramping rate limits of generating units, (17) is the power flow limit for each transmission line, (18) ensures the power balance for each bus.

\subsubsection{Day-ahead operation constraints of DHS}

As shown in Fig. 1, the DHS consists of heat sources, supply pipelines, return pipelines, and end-users. The EPS is coupled with the DHS through CHP units and EBs. EBs are regarded as electric loads, which consume the electricity to generate heat. In this paper, the DHS is operated under the constant flow and variable temperature (CF-VT) control strategy.

$$
H_{h, t}^{\mathrm{CHP}}=\eta_{h}^{\mathrm{CHP}} P_{h, t}^{\mathrm{CHP}}, \forall h \in \Phi_{s}^{\mathrm{CHP}}, \forall t \in T
$$

$$
H_{i, t}^{\mathrm{EB}}=\eta_{i}^{\mathrm{EB}} P_{i, t}^{\mathrm{EB}}, \forall i \in \Phi_{s}^{\mathrm{EB}}, \forall t \in T
$$

$$
\sum_{h \in \Omega_{j}^{\mathrm{CHP}}} H_{h, t}^{\mathrm{CHP}}+\sum_{i \in \Omega_{j}^{\mathrm{EB}}} H_{i, t}^{\mathrm{EB}}=c \cdot m_{j, t}^{\mathrm{HS}} \cdot\left(\tau_{n d, t}^{\mathrm{S}, \mathrm{ND}}-\tau_{n d, t}^{\mathrm{R}, \mathrm{ND}}\right) \forall j \in \Phi_{s}^{\mathrm{HS}}, n d=\Omega_{j}^{\mathrm{HN}}, \forall t \in T
$$

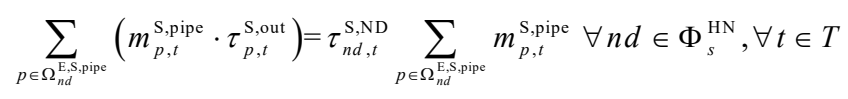

$$
\sum_{p \in \Omega_{n, d}^{\text {R., pipe }}}\left(m_{p, t}^{\mathrm{R}, \text { pipe }} \cdot \tau_{p, t}^{\mathrm{R}, \text { out }}\right)=\tau_{n d, t}^{\mathrm{R}, \mathrm{ND}} \sum_{p \in \Omega_{n d}^{\mathrm{ER}, \text { pipe }}} m_{p, t}^{\mathrm{R} \text {,pipe }} \forall n d \in \Phi_{s}^{\mathrm{HN}}, \forall t \in T
$$

$$
\tau_{p, t}^{\mathrm{S}, \mathrm{in}}=\tau_{n d, t}^{\mathrm{S}, \mathrm{ND}}, \forall n d \in \Phi_{s}^{\mathrm{HN}}, \forall p \in \Phi_{s}^{\mathrm{BS}, \text { pipe }}, \forall t \in T
$$

$$
\tau_{p, t}^{\mathrm{R} \text {,in }}=\tau_{n d, t}^{\mathrm{R}, \mathrm{ND}}, \forall n d \in \Phi_{s}^{\mathrm{HN}}, \forall p \in \Phi_{s}^{\mathrm{B}, \mathrm{R}, \mathrm{pipe}}, \forall t \in T
$$

$$
H_{l, t}^{\mathrm{HD}}=c \cdot m_{l, t}^{\mathrm{HD}} \cdot\left(\tau_{n d, t}^{\mathrm{S}, \mathrm{ND}}-\tau_{n d, t}^{\mathrm{R}, \mathrm{ND}}\right) \forall l \in \Phi_{s}^{\mathrm{HD}}, n d=\Omega_{l}^{\mathrm{HN}}, \forall t \in T
$$

$$
0 \leq H_{i, t}^{\mathrm{EB}} \leq H_{i}^{\mathrm{EB}, \max }, \forall i \in \Phi_{s}^{\mathrm{EB}}, \forall t \in T
$$

Eq. (19) is the coupling function of back-pressure CHP units, (20) denotes the relationship between heat generation and power consumption of EBs, (21) is the calculation of the heat energy provided by heat sources, (22)-(23) describe the temperature drop in the supply and return pipelines caused by heat losses, (24)-(25) are the temperature mixture of each node according to the energy conservation law, (24)-(27) mean that the temperature of pipelines flowing out of one node is equal to the mixed temperature at this node, (28) is the model of heat demands, and (29) is the capacity constraint of EBs.

Since part of the operational constraints in the second stage are similar to the corresponding constraints in the first stage, inequalities (15)-(17) and (29) are written in a compact form as in (30), and equalities (18)-(28) are written as constraint (31) to simplify the expression in the following sections. 


$$
L I_{s}^{\mathrm{DA}}\left(P_{h, t}^{\mathrm{CHP}}, P_{g, t}^{\mathrm{G}}, P_{i, t}^{\mathrm{EB}}, \delta_{s, n, t}\right) \leq 0
$$

$$
L E_{s}^{\mathrm{DA}}\left(H_{h, t}^{\mathrm{CHP}}, H_{i, t}^{\mathrm{EB}}, \tau_{n d, t}^{\mathrm{S} / \mathrm{ND}}, \tau_{p, t}^{\mathrm{S} / \mathrm{R}, \mathrm{in}}, \tau_{p, t}^{\mathrm{S} R \text {,out }}\right)=0
$$

\subsection{Real-time regulation constraints of EPS and DHS}

The second-stage problem is to adjust the outputs of non-wind generating units in the real-time operation to cope with wind power deviations. Once the uncertainty of wind power is revealed, CHP units, thermal units, and EBs regulate their outputs according to the day-ahead energy and reserve scheduling. The wind uncertainty set is represented by a box in this paper.

$\mathbf{U}_{s}=\left\{\Delta \mathbf{W} \mid-\Delta W_{w, t}^{\max } \leq \Delta W_{w, t} \leq \Delta W_{w, t}^{\max }, \forall w \in \Phi_{s}^{\mathrm{WF}}, \forall t\right\}$

$$
0 \leq r_{h, t}^{\mathrm{CHP}, \mathrm{U}} \leq R_{h, t}^{\mathrm{CHP}}, \forall h \in \Phi_{s}^{\mathrm{CHP}}, \forall t \in T
$$$$
0 \leq r_{h, t}^{\mathrm{CHP}, \mathrm{D}} \leq R_{h, t}^{\mathrm{CHP}}, \forall h \in \Phi_{s}^{\mathrm{CHP}}, \forall t \in T
$$$$
0 \leq r_{g, t}^{\mathrm{G}, \mathrm{U}} \leq R_{g, t}^{\mathrm{G}}, \forall g \in \Phi_{s}^{\mathrm{G}}, \forall t \in T
$$$$
0 \leq r_{g, t}^{\mathrm{G}, \mathrm{D}} \leq R_{g, t}^{\mathrm{G}}, \forall g \in \Phi_{s}^{\mathrm{G}}, \forall t \in T
$$$$
0 \leq W_{w, t}^{\mathrm{spill}} \leq P_{w, t}^{\mathrm{wd}}+\Delta W_{w, t}, \forall w \in \Phi_{s}^{\mathrm{wF}}, \forall t \in T
$$

$$
=\sum_{m \in \Omega_{n}^{\mathrm{N}}} B_{n m}\left(\delta_{s, n, t}^{\mathrm{RT}}-\delta_{s, m, t}^{\mathrm{RT}}-\delta_{s, n, t}+\delta_{s, m, t}\right), \forall n \in \Phi_{s}^{\mathrm{N}}, \forall t \in T
$$

$$
L I_{s}^{\mathrm{RT}}\left(P_{h, t}^{\mathrm{CHP}, \mathrm{RT}}, P_{g, t}^{\mathrm{G}, \mathrm{RT}}, P_{i, t}^{\mathrm{EB}, \mathrm{RT}}, \delta_{s, n, t}\right) \leq 0
$$

$$
L E_{s}^{\mathrm{RT}}\left(H_{h, t}^{\mathrm{CHP}, \mathrm{RT}}, H_{i, t}^{\mathrm{EB}, \mathrm{RT}}, \tau_{n d, t}^{\mathrm{SR}, \mathrm{ND}, \mathrm{RT}}, \tau_{p, t}^{\mathrm{S} R \mathrm{in}, \mathrm{RT}}, \tau_{p, t}^{\mathrm{SR}, \mathrm{ou}, \mathrm{RT}}\right)=0
$$

Eq. (32) represents the wind power uncertainty set, which is a polyhedron, (33)-(36) indicate that the redispatch of CHP units and thermal units is not greater than the reserve scheduled in the day-ahead operation, (37) means that the wind spillage is not greater than the real wind generation, (38) is the limit of load shedding, (39) preserves the power balance at each bus in the realtime operation, and (40)-(41) are real-time operation constraints similar to the day-ahead constraints (30)-(31).

\section{Solution methodology}

In this section, the mathematical formulation of the decentralized robust energy and reserve optimization in Section 3 is written in a compact form firstly. Then, an improved ADMM algorithm with adaptive penalty parameters is adopted to achieve the independent yet coordinated operation for multiple IEHSs with limited exchanged information. Finally, the column-and-constraint generation $(\mathrm{C} \& \mathrm{CG})$ algorithm is adopted to solve the tri-level robust optimization for each IEHS addressing the wind uncertainty. 


\subsection{Compact formulation of decentralized two-stage robust optimization}

For a concise expression in the following discussion, the mathematical model of the decentralized two-stage robust optimization for each IEHS is written in a compact matrix form as follows.

$$
\begin{gathered}
\min _{\mathbf{x}_{s}, \boldsymbol{\delta}_{s}}\left(\mathbf{c}_{s}^{\mathrm{T}} \mathbf{x}_{s}+\boldsymbol{\lambda}_{s}^{\mathrm{T}}\left(\boldsymbol{\delta}_{s}-\overline{\boldsymbol{\delta}}\right)+0.5 \rho\left\|\boldsymbol{\delta}_{s}-\overline{\boldsymbol{\delta}}\right\|_{2}^{2}+\max _{\mathbf{u}_{s} \in \mathbf{U}_{s} \mathbf{y}_{s} \in F\left(\mathbf{x}_{s}, \mathbf{u}_{s}\right)} \mathbf{d}_{s}^{\mathrm{T}} \mathbf{y}_{s}\right) \\
\text { s.t. } \quad \mathbf{A}_{s}\left(\mathbf{x}_{s}+\boldsymbol{\delta}_{s}\right) \leq \mathbf{b}_{s}, \mathbf{B}_{s}\left(\mathbf{x}_{s}+\boldsymbol{\delta}_{s}\right)=\mathbf{a}_{s}, \mathbf{x}_{s} \in \mathbb{R}_{+}^{\mathrm{n}} \\
F\left(\mathbf{x}_{s}, \mathbf{u}_{s}\right)=\left\{\mathbf{y}_{s} \mid \mathbf{C}_{s} \mathbf{y}_{s}+\mathbf{D}_{s} \mathbf{x}_{s} \leq \mathbf{g}_{s}\right. \\
\mathbf{G}_{s} \mathbf{y}_{s}+\mathbf{M}_{s} \mathbf{u}_{s}=\mathbf{h}_{s} \\
\left.\mathbf{y}_{s} \in \mathbb{R}_{+}^{\mathrm{n}}\right\}
\end{gathered}
$$

where $\mathbf{x}_{s}$ is the vector of first-stage decision variables representing the day-ahead energy and reserve scheduling, $\boldsymbol{\delta}_{s}$ is the vector of boundary buses' phase angles, $\mathbf{y}_{\mathbf{s}}$ is the decision vector in the second-stage representing redisptach variables, and $\mathbf{u}_{s}$ is the uncertain variables, $F\left(\mathbf{x}_{\mathbf{s}}, \mathbf{u}_{s}\right)$ is the admissible redispatch set in the real-time operation. Eq. (43) includes all day-ahead operation constraints (9)-(29). Eq. (46) shows $\mathbf{y}_{s}$ is positive.

\subsection{Improved locally adaptive ADMM decentralized algorithm}

In this paper, the ADMM algorithm is chosen to implement the decentralized energy and reserve optimization for multiple IEHSs. The convergence performance of the standard ADMM is sensitive to the value of penalty parameter $\rho$. In [28], the penalty parameter in the standard ADMM is fixed and the convergence is slow. In order to accelerate the convergence rate, some variants of ADMM with adaptive penalty parameters are studied. Ref. [29] proposed to update the penalty parameter to balance the magnitudes of the primal and dual residuals, whereas the convergence rate of the ADMM is not guaranteed. In [30], it was proved that the lower iteration complexity of the improved ADMM (LA-ADMM) was adaptive to the local sharpness property of the objective function, and it is adopted in this paper to reduce the iteration and computation time.

In the LA-ADMM, the original iterative coordination process between separated subsystems in the standard ADMM is decomposed into multiple stages with the updated penalty parameter. At each stage $j$, the standard ADMM is performed with a fixed penalty parameter $\rho^{j}$ and is finished within a constant number of iterations $K$. In each iteration, the optimization is performed separately with the updated Lagrangian multipliers and the exchanged phase angles of boundary buses fixed in the previous iteration, which is shown as bellow.

$$
\min _{\mathbf{x}_{s}^{(k)}, \mathbf{\delta}_{s}^{(k)}}\left\{\mathbf{c}_{s}^{\mathrm{T}} \mathbf{x}_{s}^{(k)}+\left(\lambda_{s}^{(k)}\right)^{\mathrm{T}}\left(\boldsymbol{\delta}_{s}^{(k)}-\overline{\boldsymbol{\delta}}^{(k-1)}\right)+0.5 \rho^{j}\left\|\boldsymbol{\delta}_{s}^{(k)}-\overline{\boldsymbol{\delta}}^{(k-1)}\right\|_{2}^{2}+\max _{\mathbf{u}_{s} \mathbf{U}_{s} \mathbf{y}_{s}^{(k)} \in F_{s}\left(\mathbf{x}_{s}^{(k)}, \mathbf{u}_{s}\right)} \mathbf{d}_{s}^{T} \mathbf{y}_{s}^{(k)}\right\}
$$

$$
\text { s.t. (43)-(46) }
$$

After obtaining the optimal results $\boldsymbol{\delta}_{s}^{(k)}$, the Lagrangian multipliers are updated as follows,

$$
\boldsymbol{\lambda}_{s}^{(k)}=\boldsymbol{\lambda}_{s}^{(k-1)}+\rho^{j}\left(\boldsymbol{\delta}_{s}^{(k)}-\overline{\boldsymbol{\delta}}^{(k)}\right)
$$

Once each stage with the standard ADMM is finished, the penalty parameter $\rho$ is updated by multiplying a constant factor larger than 1 and used in the next stage as a parameter. In the LA-ADMM, the penalty parameter is allowed to increase to infinity. In the end, the whole iteration process is finished when the penalty term in each IEHS reaches an agreement, i.e., the maximum value of the primal residual $\Delta \boldsymbol{\delta}_{s}^{\text {prim, }(k)}$ and the dual residual $\Delta \boldsymbol{\delta}_{s}^{\text {dual, }(k)}$ is less than the convergence tolerance $\varepsilon$, which is expressed as follows. 


$$
\max \left\{\triangle \boldsymbol{\delta}_{s}^{\text {prim, },(k)}, \triangle \boldsymbol{\delta}_{s}^{\text {dual },(k)}\right\} \leq \varepsilon
$$

The solution procedure of the LA-ADMM is described as follows.

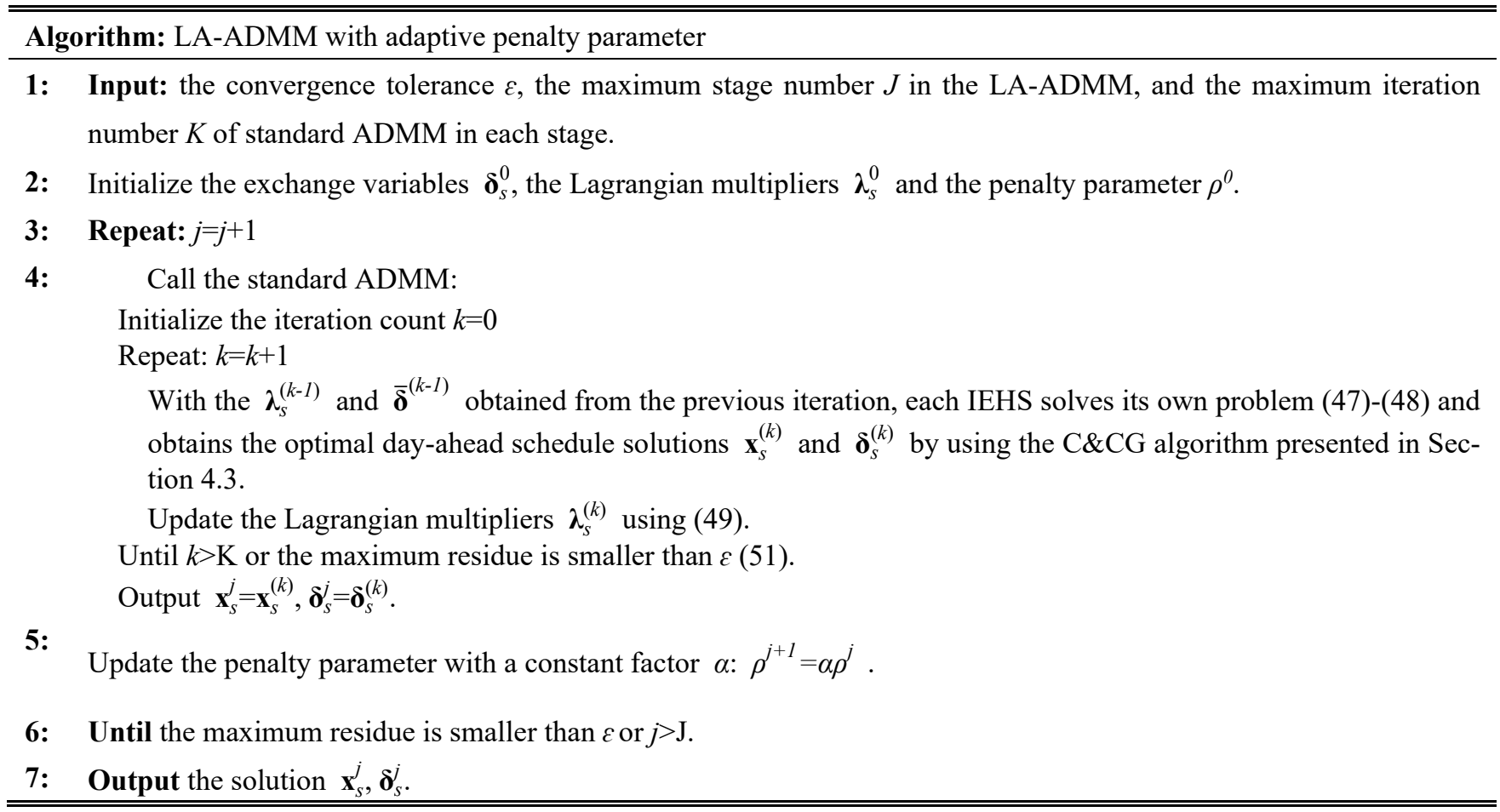

\subsection{C\&CG algorithm for each IEHS}

Based on the decentralized framework, the C\&CG algorithm is adopted to solve the robust energy and reserve optimization (47)-(48) for each IEHS [31]. Firstly, the tri-level problem is decomposed into a master problem (MP) and a subproblem (SP). Then, the MP and SP are solved iteratively. In each iteration, the constraints corresponding to the scenario identified in the SP are generated and added to the MP. The MP is to minimize the total system operation cost with introducing the cutting planes in each iteration. The max-min SP is to optimize the real-time redispatch cost under the worst-case realization of wind power uncertainty. Since the model of the inner min problem is linear, the strong duality holds and the max-min SP is reformulated using KKT conditions. The iteration process converges when the difference between the objective function value of the MP and SP is smaller than the convergence tolerance.

\section{Simulation results}

This section presents the case studies to demonstrate the effectiveness of the proposed decentralized adaptive robust energy and reserve co-optimization scheme. The topology of the test system consists of three IEHSs connected by tie-lines, which is shown in Fig. 3. Each IEHS consists of a modified six-bus EPS and a four-node DHS, coupled by CHP units and EBs. In the whole system, there are three CHP units, three EBs, four thermal power units, three wind farms and four electric demands. The parameters of generators are listed in Table 1. The cost of load shedding is set as $200 \$ / \mathrm{MWh}$. In the DHS, the CF-VT control strategy is used. In order to ensure the quality of heat supply, the supply temperature of heat sources is set as $80^{\circ} \mathrm{C}$. In the supply heating networks, the temperature of pipelines is between $60-80^{\circ} \mathrm{C}$, and the temperature in the return heating networks depends on the heat demands. The hourly electric and heat loads in the whole system are shown in Fig. 4. In the decentralized method, the initial value of penalty 
parameter $\rho^{0}$ and the constant factor to update $\rho$ is set as 0.5 and 3, respectively. The convergence tolerance of the LA-ADMM is set as 0.001. The case studies were carried out in General Algebraic Modeling System (GAMS) using the CPLEX as the QCP solver.
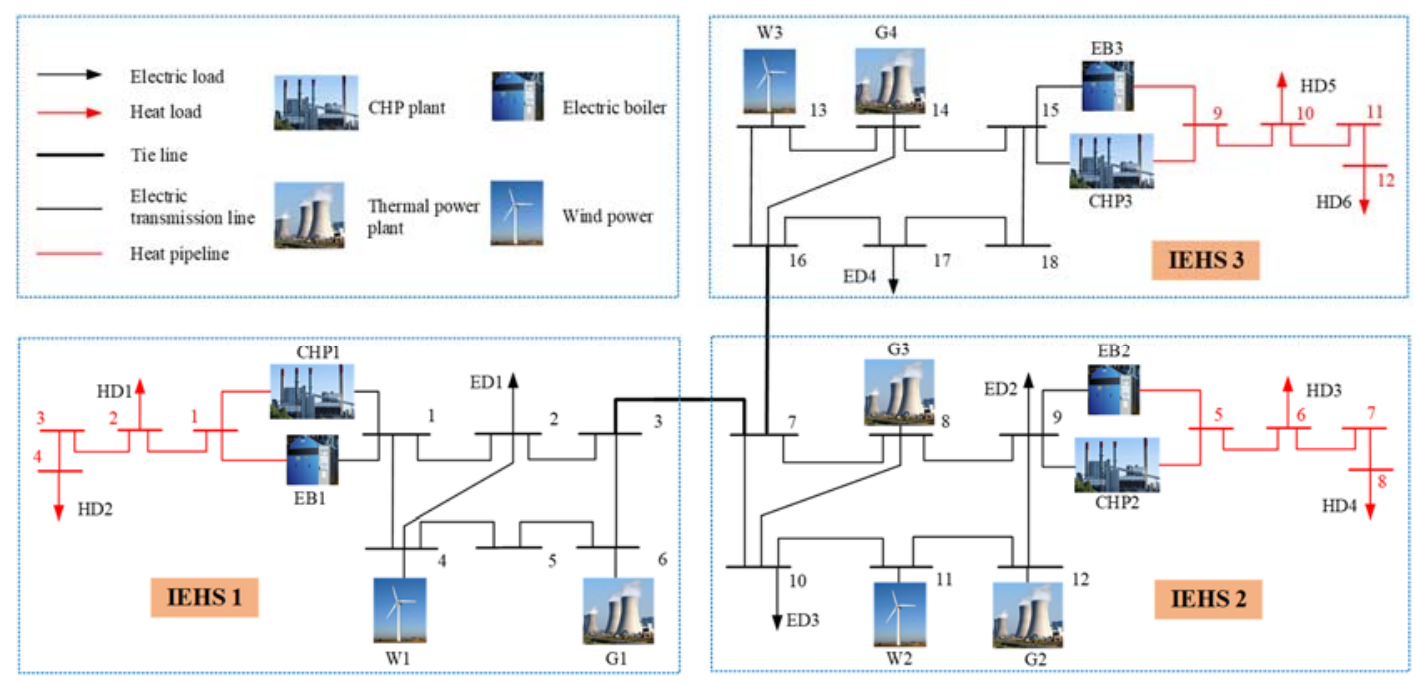

Fig. 3. Test system with three IEHSs

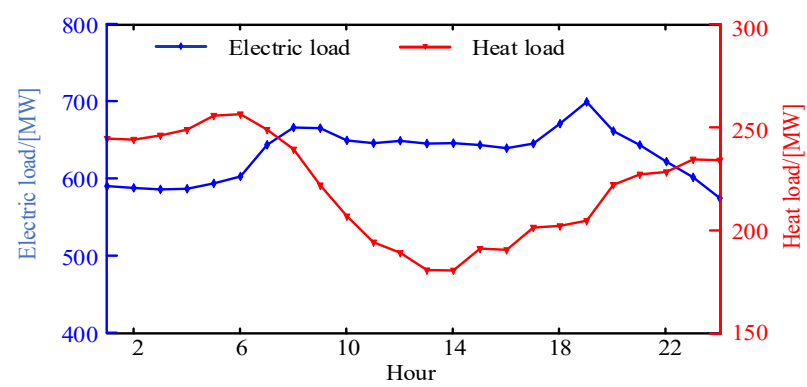

Fig. 4. Hourly electric load and heat load in the whole system

Table 1 Parameters of generators

\begin{tabular}{ccccccc}
\hline \hline Energy generators & CHP 1/2/3 & G1 & G2 & G3 & G4 & EB 1/2/3 \\
\hline Capacity $(\mathrm{MW})$ & 200 & 150 & 100 & 100 & 110 & 12 \\
Ramp rate $(\mathrm{MW} / \mathrm{h})$ & 30 & 50 & 50 & 50 & 50 & $/$ \\
Energy price $(\$ / \mathrm{MWh})$ & 16 & 15 & 10 & 15 & 12 & $/$ \\
Reserve price $(\$ / \mathrm{MW})$ & 10 & 12 & 12 & 6 & 10 & $/$ \\
Efficiency & 1.5 & $/$ & $/$ & $/$ & $/$ & 1 \\
\hline \hline
\end{tabular}

\subsection{Validation of proposed two-stage robust energy and reserve co-optimization}

In this subsection, the traditional single-stage optimization is considered as a comparative method to illustrate the effectiveness of the proposed adaptive robust energy and reserve optimization model with the utilization of the reserve capacity from the DHS.

(1) Conventional single-stage optimization with the pre-defined system reserve requirement [13]. The real-time redispatch is optimized after obtaining the day-ahead energy and reserve scheduling.

(2) The proposed two-stage robust optimization model. The day-ahead energy and reserve are optimized taking into account the operation constraints in the real-time stage.

Table 2 shows two comparative cases. In these cases, EBs are introduced into the DHS, enabling CHP units to adjust the outputs and provide reserve capacity to the EPS. The total system operation costs are shown in Table 2 . Fig. 5 illustrates the optimized 
day-ahead heat generation and reserve allocation in Cases 1 and 2 under the wind uncertainty, as well as the real reserve deployment in the second stage.

Table 2 Study cases

\begin{tabular}{cccc}
\hline \hline Cases & CHP as reserve & EB & Optimized method \\
\hline Case 1 & $\sqrt{ }$ & $\sqrt{ }$ & Conventional single-stage model \\
Case 2 & $\sqrt{ }$ & $\sqrt{ }$ & Proposed two-stage robust model \\
\hline \hline
\end{tabular}

Case 1: In this case, the conventional single-stage optimization is adopted to optimize the day-ahead energy and reserve scheduling and CHP units are assumed able to provide full reserve capacity as thermal units. The optimal day-ahead heat energy and reserve scheduling are presented in Fig. 5(a). It shows that, in the day-ahead stage, both CHP units and thermal units are scheduled to provide reserve capacities for the EPS. CHP units, with cheaper reserve prices, are scheduled first. However, as shown in the "Real reserve deployment" sub-figure in Fig. 5(a), the available balancing resources in the real-time operation are insufficient to cope with the uncertainty. When the EPS needs upward regulation in real-time operation, CHP units cannot provide the same upward regulation as scheduled in the day-ahead stage. It means the reserve scheduled in the day-ahead stage is not actually available. As can be seen from the "Heat scheduling" sub-figure in Fig. 5(a), EBs are not scheduled to provide heat in the dayahead stage and hence CHP units cannot change their outputs to keep the heat balance. Therefore, part of electric loads have to be shed, which results in the high redispatch cost $\$ 192,372.00$, as shown in Table 3. This is because the conventional single-stage optimization does not consider the real-time operation constraints of CHP units, which are constrained by the heat demand.

Table 3 Total system costs of different study cases

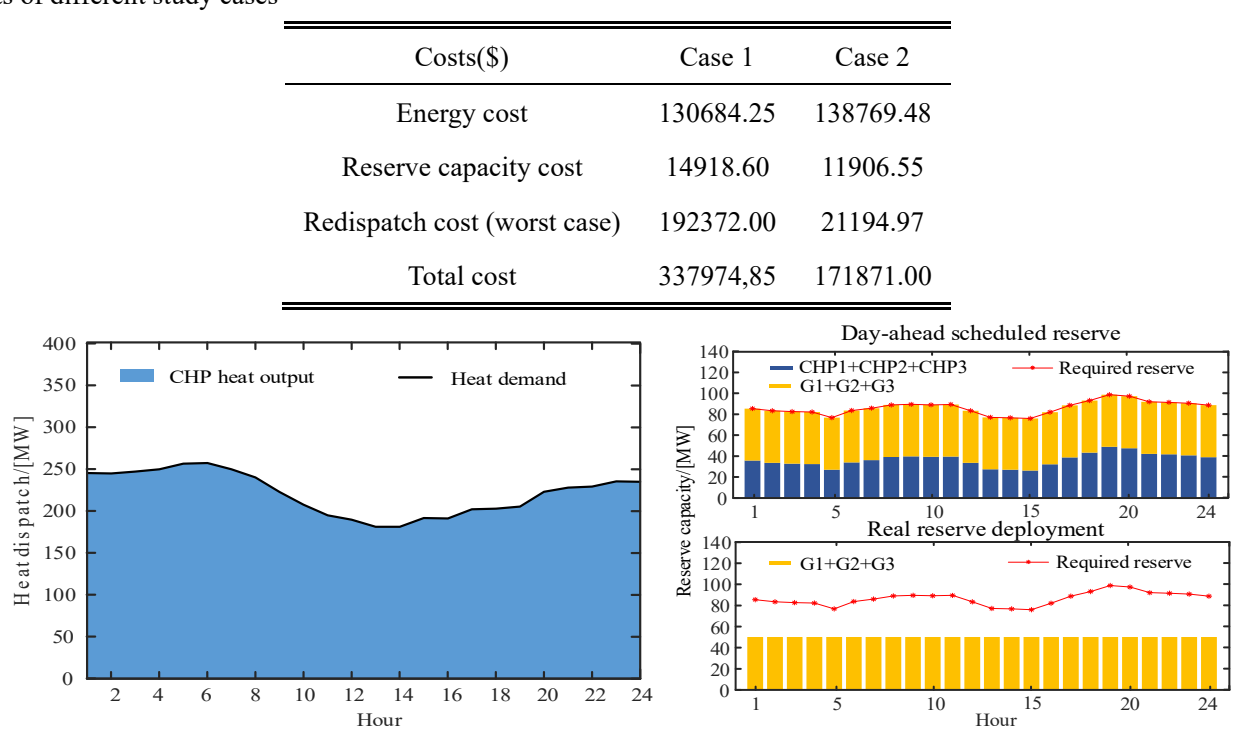

(a) Day-ahead schedule of heat energy and reserve capacity in Case 1
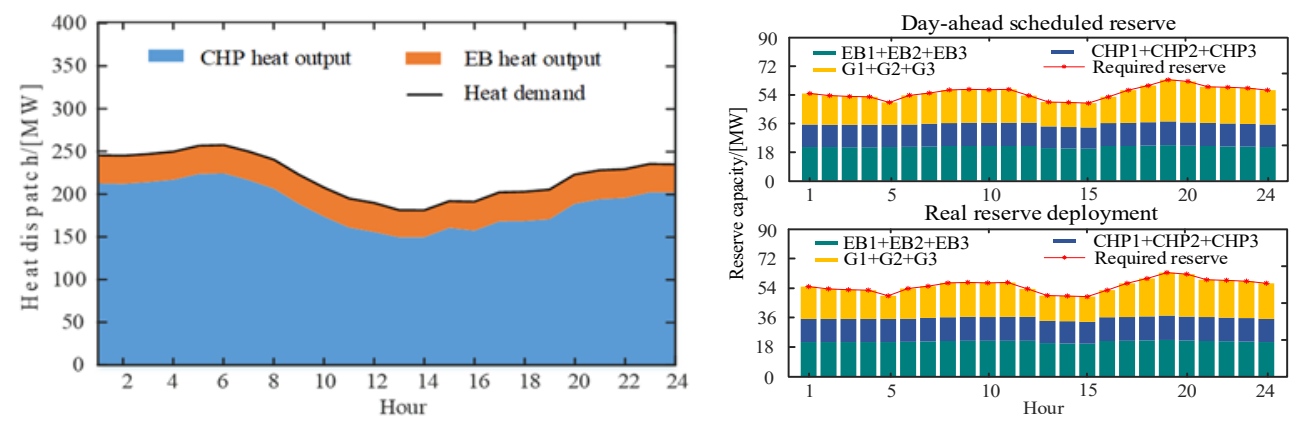

(b) Day-ahead schedule of heat energy and reserve capacity in Case 2 
Fig. 5. Optimal results of day-ahead heat schedule and reserve allocation

Case 2: In this case, the proposed two-stage robust optimization method is used for the day-ahead energy and reserve scheduling, which accounts for the real-time operation constraints of both the EPS and DHS. Fig. 5(b) shows the day-ahead heat energy and reserves scheduling in this case. It can be seen that EBs are scheduled to provide heat in the day-ahead stage, which enabling CHP units to regulate their outputs to some extent. Compared with the optimal results in Case 1, the "Real reserve deployment" subfigure in Fig. 5(b) shows that the reserve capacity scheduled in Case 2 is feasible and sufficient for the real-time operation, which ensures the secure and reliable operation of multiple IEHSs under the wind uncertainty. Moreover, the "Day-ahead scheduled reserve" sub-figure in Fig. 5(b) shows that both CHP units and EBs provide reserve capacity to the EPS, of which the reserve price is lower than that of thermal units. Thus, compared with Case 1, the reserve capacity cost in Case 2 decreases from $\$ 14,918.60$ to $\$ 11,906.55$, as illustrated in Table 3. It indicates that, with adopting the two-stage adaptive robust optimization, the reserve capacity provided by the DHS are utilized in a more proper and reliable way and the economic efficiency of reserve allocation is improved as well. Since EBs are scheduled to supply heat instead of CHP units and it is not as efficient as CHP units, the energy cost in Case 2 increases a bit compared with Case 1. Even so, the overall operation cost of the whole system in Case 2 is reduced.

\subsection{Comparison of system operation costs under three different operation frameworks}

To demonstrate the economic benefits of the coordinated energy and reserve optimization among multiple IEHSs and the validity of the decentralized algorithm, the day-ahead energy and reserve scheduling of multiple IEHSs is optimized under isolated, centralized and ADMM-based decentralized operation frameworks. Under the centralized and decentralized operation, IEHSs are operated in a coordinated way. All the operations are determined with the proposed two-stage robust optimization. Table 4 compares the total operation costs under three different operation frameworks. Fig. 6 shows the load shedding of electric demand ED4 in real-time stage under isolated and the optimal power flows of tie-lines in three IEHSs under the coordinated operation, which are scheduled 24 hours ahead.

Table 4 Compared system costs of different operation frameworks

\begin{tabular}{cccc}
\hline \hline Costs $(\$)$ & Isolated & Centralized & Decentralized \\
\hline Energy cost & 140590.44 & 138769.48 & 138769.56 \\
Reserve capacity cost & 10887.51 & 11906.55 & 11906.55 \\
Redispatch cost (worst case) & 70924.09 & 21194.97 & 21194.80 \\
Total cost & 222402.04 & 171871.00 & 171870.91 \\
\hline \hline
\end{tabular}

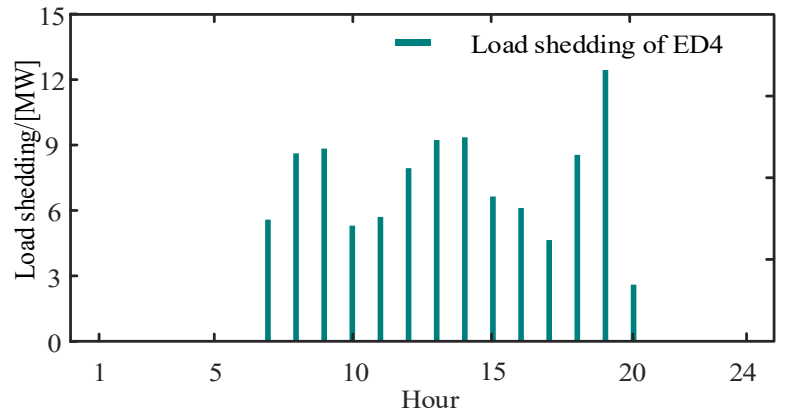

(a) Load shedding of G4 in real-time stage under isolated operation

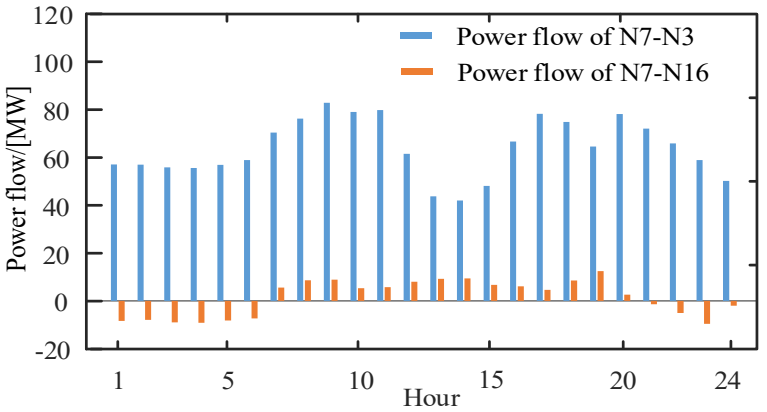

(b) Optimized tie-line power flow under decentralized operation

Fig. 6. Load shedding and tie-line power flow under different operations

In the isolated operation, IEHSs are operated separately without any power exchange or resources sharing. In IEHS 3 with the highest wind power penetration, reserves are not enough to cope with the wind uncertainty. Thus, there are $101.90 \mathrm{MW}$ of electric loads curtailed in IEHS 3 , which is shown in Fig. 6 (a). Consequently, the total operation cost of IEHSs is high, as shown in Table 
4. Under the coordinated operation with the centralized and decentralized operation strategies, multiple IEHSs are interconnected through tie-lines. It allows the cheapest resource in the whole system to be fully used. As can be seen from Fig.6 (b), on the one hand, the surplus energy with lower costs in IEHS 1 is transmitted to IEHS 2 through the tie-line N7-N3, which decreases the energy cost of the whole system by $\$ 1820.96$. On the other hand, when the reserve capacity in IEHS 3 is not enough from 7:00 to 20:00, the electricity is imported from IEHS 1 through tie-line N7-N16. Thus, the load shedding is avoided in the coordinated operation. Compared with the isolated operation, the available reserves in IEHS 3 are improved thanks to sharing resources with other IEHSs. Regarding the decentralized operation, Table 4 shows that the gap of the objective values between the decentralized and centralized operation is less than $0.001 \%$, indicating the decentralized solution is satisfactory. It means that with limited information exchange, the synergistic but independent operation for multiple IEHSs is achieved by the decentralized operation framework without losing the economic efficiency, where the privacy of different systems is preserved compared with the centralized operation.

\subsection{Performance of improved $L A-A D M M$ with adaptive penalty parameters}

In this subsection, the standard ADMM is used as a benchmark to demonstrate the effectiveness of the improved LA-ADMM. Instead of using a fixed penalty parameter in the standard ADMM, an adaptive updated penalty parameter is adopted in the LAADMM, where the lower iteration complexity has been proved theoretically [30]. Taking Case 2 as an example, the convergence processes of two decentralized algorithms, which is described by the maximum residual versus the iterations, are shown in Fig. 7. It can be seen that, along with the increase of iterations, the residual of two decentralized methods both decreases. In the end, the algorithm is converged when the maximum residual is lower than the convergence tolerance, implying that the power flow of the tie-line connecting adjacent IEHSs reaches an agreement. Fig. 7 shows that every time the penalty parameter is updated, the maximum residual in the LA-ADMM decreases a lot more than that in the standard ADMM. The LA-ADMM-based decentralized operation converged before 24 iterations, whereas the iteration of the standard-ADMM-based decentralized operation is 82 . The convergence rate of the LA-ADMM has been increased by $70 \%$ compared with the standard ADMM. As such, the total exchanged data in the whole process and the corresponding communication demand between two IEHSs are both reduced by using the improved LA-ADMM.

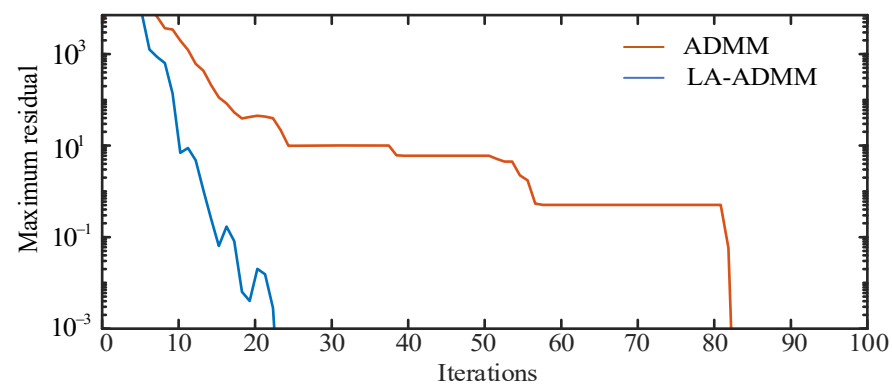

Fig. 7. Residual in each iteration with different ADMM algorithms

Table 5 Compared costs of different ADMM algorithms

\begin{tabular}{ccc}
\hline \hline Costs $(\$)$ & Standard ADMM & LA-ADMM \\
\hline Energy cost & 138769.47 & 138769.56 \\
Reserve capacity cost & 11906.55 & 11906.55 \\
Redispatch cost (worst case) & 21194.95 & 21194.80 \\
Total cost & 171870.97 & 171870.91 \\
\hline \hline
\end{tabular}

Furthermore, the optimal operation costs in Case 2 using the standard ADMM and improved LA-ADMM are shown in Table 
that the LA-ADMM can improve the computation efficiency while achieving a satisfying operation result as the standard ADMM.

\section{Conclusion}

This paper proposes a decentralized robust energy and reserve co-optimization scheme for multiple IEHSs by using the available reserve capacity provided by the DHS and resources sharing among multiple IEHSs. For each IEHS, the two-stage adaptive robust optimization considers the redispatch constraints of both the EPS and DHS and guarantees the feasibility of reserve schedules to cope with the wind uncertainty. A decentralized algorithm based on the ADMM is adopted to preserve the information privacy of different entities, and hence multiple IEHSs are operated in a synergistic but independent way. Simulation results show thatthe available reserve capacity is increased and the energy cost of the whole system is reduced by sharing resources among multiple IEHSs. The gap of the objective values between the decentralized operation and centralized operation is less than $0.001 \%$, which validates the effectiveness of the proposed decentralized operation for multiple IEHSs. Compared to the standard ADMM, the convergence of the LA-ADMM algorithm with adaptive penalty parameters is increased by $70 \%$.

\section{Acknowledgements}

The PhD student, Jin Tan, is jointly supported by the China Scholarship Council (CSC) (No. 201806270242) and Technical University of Denmark (DTU). This work was also financially supported by the Innovationsfonden through the project "Using flexible district heating with heat pumps for integrated electricity and heat dispatch with renewables" (HEAT4RES), and by the Ministry of Science and Technology (MOST) China through the project (2018YFE0106600).

\section{References}

[1] Xu Q, Zhang N, Kang C, Xia Q, He D, Liu C, et al. A Game Theoretical Pricing Mechanism for Multi-Area Spinning Reserve Trading Considering Wind Power Uncertainty. IEEE Trans Power Syst 2016;31:1084-95.

[2] Lund H. Large-scale integration of wind power into different energy systems. Energy 2005;30(13):2402-12.

[3] Tan J, Wu Q, Hu Q, Wei W, Liu F. Adaptive robust energy and reserve co-optimization of integrated electricity and heating system considering wind uncertainty. Appl Energy 2020;260:114230.

[4] Li Z, Wu W, Shahidehpour M, Wang J, Zhang B. Combined Heat and Power Dispatch Considering Pipeline Energy Storage of District Heating Network. IEEE Trans Sustain Energy 2015;7:12-22.

[5] Cai H, Ziras C, You S, Li R, Honoré K, Bindner HW. Demand side management in urban district heating networks. Appl Energy 2018;230:506-18.

[6] Liu B, Meng K, Dong ZY, Wei W. Optimal Dispatch of Coupled Electricity and Heat System with Independent Thermal Energy Storage. IEEE Trans Power Syst 2019;34:3250-63.

[7] Papaefthymiou G, Hasche B, Nabe C. Potential of heat pumps for demand side management and wind power integration in the German electricity market. IEEE Trans Sustain Energy 2012;3:636-42.

[8] Zhang N, Lu X, McElroy MB, Nielsen CP, Chen X, Deng Y, et al. Reducing curtailment of wind electricity in China by employing electric boilers for heat and pumped hydro for energy storage. Appl Energy 2016;184:987-94.

[9] Lu S, Gu W, Zhou S, Yu W, Yao S, Pan G. High Resolution Modeling and Decentralized Dispatch of Heat and Electricity Integrated Energy System. IEEE Trans Sustain Energy 2019;PP:1-1.

[10] Lin C, Wu W, Zhang B, Sun Y. Decentralized Solution for Combined Heat and Power Dispatch Through Benders Decomposition. IEEE Trans Sustain Energy 2017;8:1361-72.

[11] Huang J, Li Z, Wu QH. Coordinated dispatch of electric power and district heating networks: A decentralized solution using optimality condition decomposition. Appl Energy 2017;206:1508-22. 
[12] Caramanis M, Ntakou E, Hogan WW, Chakrabortty A, Schoene J. Co-optimization of power and reserves in dynamic T\&D power markets with nondispatchable renewable generation and distributed energy resources. Proc IEEE 2016;104:807-36.

[13] Wang J, Zhong H, Xia Q, Kang C, Du E. Optimal joint-dispatch of energy and reserve for CCHP-based microgrids. IET Gener Transm Distrib 2016;11:78594.

[14] Li G, Zhang R, Jiang T, Chen H, Bai L, Cui H, et al. Optimal dispatch strategy for integrated energy systems with CCHP and wind power. Appl Energy 2017;192:408-19.

[15] Pan Z, Guo Q, Sun H. Feasible region method based integrated heat and electricity dispatch considering building thermal inertia. Appl Energy 2017;192:395407.

[16] Zhou Y, Hu W, Min Y, Dai Y. Integrated power and heat dispatch considering available reserve of combined heat and power units. IEEE Trans Sustain Energy 2019;10:1300-10.

[17] Arroyo JM, Galiana FD. Energy and Reserve Pricing in Security and Network-Constrained Electricity Markets. IEEE Trans Power Syst 2005;20:634-43.

[18] Vrakopoulou M, Margellos K, Lygeros J, Andersson G. A Probabilistic Framework for Reserve Scheduling and N - 1 Security Assessment of Systems with High Wind Power Penetration. IEEE Trans Power Syst 2013;28:3885-96.

[19] Zhang M, Ai X, Fang J, Yao W, Zuo W, Chen Z, et al. A systematic approach for the joint dispatch of energy and reserve incorporating demand response. Appl Energy 2018;230:1279-91.

[20] Ahmadi-Khatir A, Conejo AJ, Cherkaoui R. Multi-area unit scheduling and reserve allocation under wind power uncertainty. IEEE Trans Power Syst 2014;29:1701-10.

[21] Wei W, Liu F, Mei S, Hou Y. Robust energy and reserve dispatch under variable renewable generation. IEEE Trans Smart Grid 2015;6:369-80.

[22] Zugno M, Conejo AJ. A robust optimization approach to energy and reserve dispatch in electricity markets. Eur J Oper Res 2015;247:659-71.

[23] Kargarian A, Mohammadi J, Guo J, Chakrabarti S, Barati M, Hug G, et al. Toward Distributed/Decentralized DC Optimal Power Flow Implementation in Future Electric Power Systems. IEEE Trans Smart Grid 2018;9:2574-94.

[24] Li Z, Shahidehpour M, Wu W, Zeng B, Zhang B, Zheng W. Decentralized Multiarea Robust Generation Unit and Tie-Line Scheduling under Wind Power Uncertainty. IEEE Trans Sustain Energy 2015;6:1377-88.

[25] He Y, Yan M, Shahidehpour M, Li Z, Guo C, Wu L, et al. Decentralized Optimization of Multi-Area Electricity-Natural Gas Flows Based on Cone Reformulation. IEEE Trans Power Syst 2018;33:4531-42.

[26] Mhanna S, Verbic G, Chapman AC. Adaptive admm for distributed ac optimal power flow. IEEE Trans Power Syst 2019;34:2025-35.

[27] https://www.pjm.com/directory/manuals/m11/index.html\#Sections/Section\%201\%20Overview\%20of\%20Energy\%20\%20Ancillary\%20Services\%20Market\%20Operations.html

[28] Boyd S, Parikh N, Chu E, Peleato B, Eckstein J. Distributed optimization and statistical learning via the alternating direction method of multipliers. Found Trends Mach Learn 2010;3:1-122.

[29] He BS, Yang H, Wang SL. Alternating direction method with self-adaptive penalty parameters for monotone variational inequalities. J Optim Theory Appl 2000;106:337-56.

[30] Xu Y, Liu M, Lin Q, Yang T. ADMM without a Fixed Penalty Parameter: Faster Convergence with New Adaptive Penalization. Adv Neural Inf Process Syst 2017;2017-Decem:1268-78.

[31] Zeng B, Zhao L. Solving two-stage robust optimization problems using a column-and- constraint generation method. Oper Res Lett 2013;41:457-61. 